\title{
Assessing Variation in the Cost of Palivizumab for Respiratory Syncytial Virus Prevention in Preterm Infants
}

\author{
Ahva Shahabi ${ }^{1} \cdot$ Desi Peneva $^{1} \cdot$ Devin Incerti $^{1} \cdot$ Kimmie McLaurin $^{2}$. \\ Warren Stevens ${ }^{1}$
}

Published online: 17 July 2017

(c) The Author(s) 2017. This article is an open access publication

\begin{abstract}
Background The variability in cost of palivizumab treatment, indicated for prevention of respiratory syncytial virus (RSV) infections in high-risk infants, has not been robustly estimated in prior studies. This study aimed to determine the cost variations of palivizumab from a US payer perspective for otherwise healthy preterm infants born 29-35 weeks gestational age (wGA) using infant characteristics and applied dosing regimens.

Methods Fenton Growth Charts were merged with World Health Organization Child Growth Standards to estimate preterm infant growth patterns. The merged growth chart was applied to infants who received palivizumab from a prospective, observational registry to determine future body weight using each infant's wGA and birth weight. Using quarter 3 (Q3) 2016-Q2 2017 vial cost, treatment costs at monthly dosing intervals were estimated using expected weights and averaged by age to derive expected mean 2016-2017 RSV seasonal costs per infant under various dosing scenarios.

Results Given different dosing scenarios (two to five doses), birth month, and growth patterns for preterm infants 29-35 wGA, the estimated average 2016-2017 seasonal cost of palivizumab treatment ranged from $\$ 3221$ to $\$ 12,568$.
\end{abstract}

Electronic supplementary material The online version of this article (doi:10.1007/s41669-017-0042-3) contains supplementary material, which is available to authorized users.

Ahva Shahabi

ahva.shahabi@pheconomics.com

1 Precision Health Economics, 11100 Santa Monica Blvd, Suite 500, Los Angeles, CA 90025, USA

2 AstraZeneca, One MedImmune Way, Gaithersburg, MD 20878, USA
Outpatient-only cost (excluding first dose at hospital discharge) ranged from $\$ 1733$ to $\$ 11,862$. The main drivers of costs were dosing regimen ( $74 \%$ of variance), dosing interacted with birth month (17\%), and wGA (6\%).

Conclusion The considerable variability in the average cost of palivizumab treatment for preterm infants is driven by choice of dosing regimen, wGA, and birth month. Therefore, when estimating the cost of palivizumab, it is important to consider both infant characteristics at each dose and potential dosing regimens.

\section{Key Points for Decision Makers}

Without accounting for heterogeneity in patient characteristics and potential dosing regimens, previous cost-effectiveness studies may not have accurately represented the real-world cost of palivizumab treatment.

The considerable variability in the average cost of palivizumab treatment for otherwise healthy preterm infants born 29-35 weeks gestational age is mainly driven by the total number of doses an infant receives (dosing regimen), the infant's birth month relative to the respiratory syncytial virus season, weeks gestational age at birth, and weight-for-age at each dose administration.

Future studies evaluating the cost of palivizumab should consider both weight-for-age at dosing and potential dosing regimens in order to provide more accurate assessments of palivizumab costeffectiveness. 


\section{Introduction}

Respiratory syncytial virus (RSV) is the most common cause of acute lower respiratory tract infection and hospitalization due to severe infection in young children [1]. In particular, preterm infants are at increased risk of experiencing severe RSV-related complications leading to hospitalization and increased morbidity and mortality, compared with full-term infants $[2,3]$.

Palivizumab, a humanized monoclonal antibody, is indicated for the prevention of serious lower respiratory tract disease caused by RSV in preterm and other high-risk infants [4], and can reduce overall burden of disease by preventing severe symptoms and reducing hospitalizations in high-risk populations. The American Academy of Pediatrics (AAP) provides continuously revised RSV immunoprophylaxis guidance regarding infant groups recommended to receive palivizumab, including timing of administration and number of doses [5]. Concerns regarding the cost of palivizumab have led to recommendations against its use in some Food and Drug Administration (FDA)-approved patient populations. For example, in 2009, infants born 32-34 weeks gestational age (wGA) were recommended to receive palivizumab only when younger than 3 months of age, with a maximum of three doses [6]. The 2014 AAP guidance update endorsed further restrictions in use and recommended against palivizumab for all preterm infants born 29-34 wGA without chronic lung disease or congenital heart disease [5]. This update instead recommended prophylaxis for infants born before 29 wGA, preterm infants with chronic lung disease, and certain infants with hemodynamically significant congenital heart disease in the first year of life. This study focuses on the treatment cost of otherwise healthy preterm infants born 29-35 wGA affected by the aforementioned changes to the 2014 AAP recommendations.

To date, studies examining the cost-effectiveness of palivizumab used a single estimate of infant weight, such as an average weight, and complete dosing of five doses per infant to calculate treatment costs [8-11]. As a result, costs for palivizumab reported in previous studies may not accurately reflect the real world; multiple factors not considered in these studies can influence the average cost, including changing weight for an infant between times of administration. Because the FDA-approved dosing is $15 \mathrm{mg} / \mathrm{kg}$ of body weight administered monthly throughout the RSV season for up to five doses, an infant's body weight at the time of administration is a key component. Furthermore, physicians may use different dosing regimens in clinical practice or may need to limit the number of doses per infant due to payer restrictions [7].
The heterogeneity among infants receiving palivizumab and dosing regimens contribute to variability in the cost of palivizumab. Patient heterogeneity has become an increasingly important topic in cost-effectiveness analyses over the past few years $[12,13]$, as variance in context and effectiveness has been shown to have a considerable influence on published cost-effectiveness estimates. This variability has led policymakers to consider more contextspecific approaches when assessing the value of new technologies [14]. Without accounting for heterogeneity in patient characteristics and dosing regimens, cost-effectiveness models may not accurately represent the realworld cost of palivizumab. This study aimed to assess the variations in the cost of palivizumab treatment among otherwise healthy preterm infants $29-35$ wGA for the 2016-2017 RSV season, based on infant characteristics and different dosing regimens to capture variations in guideline implementation across institutions.

\section{Methods}

This study estimated the cost of palivizumab for an otherwise healthy preterm infant 29-32 wGA at birth (discharged from the hospital 1 month after birth) or 33-35 wGA at birth (discharged within the same month of birth) with a specific set of characteristics (birth wGA and body weight) under several potential real-world dosing regimens. The examined dosing scenarios were based on birth month and the potential number of months after birth an infant could receive their last dose $(2,3,4$, or 5$)$, given that infants start receiving treatment during the RSV season (November to March of the following year). Treatment costs were estimated in the methodological steps outlined in Fig. 1 and summarized in Sects. 2.1-2.4. First, the Fenton Growth Charts [15] created for preterm infants and the World Health Organization (WHO) Child Growth Standards [16] were merged in order to produce a growth chart for preterm infants spanning $22 \mathrm{wGA}$ to 2 years of chronological age. Second, the growth chart was applied to a population of infants from a US prospective, observational registry to determine infant weights at each expected prophylactic administration (every 4 weeks). Third, the estimated body weight at subsequent weekly time intervals was used to estimate palivizumab treatment costs over time for each infant. Fourth, these expected costs were used to derive mean cost per infant under various dosing scenarios.

\subsection{Creating a Growth Chart for Preterm Infants}

Fenton Growth Charts, developed by Fenton and Kim [15], link intrauterine growth meta-analysis data from 22 to 36 


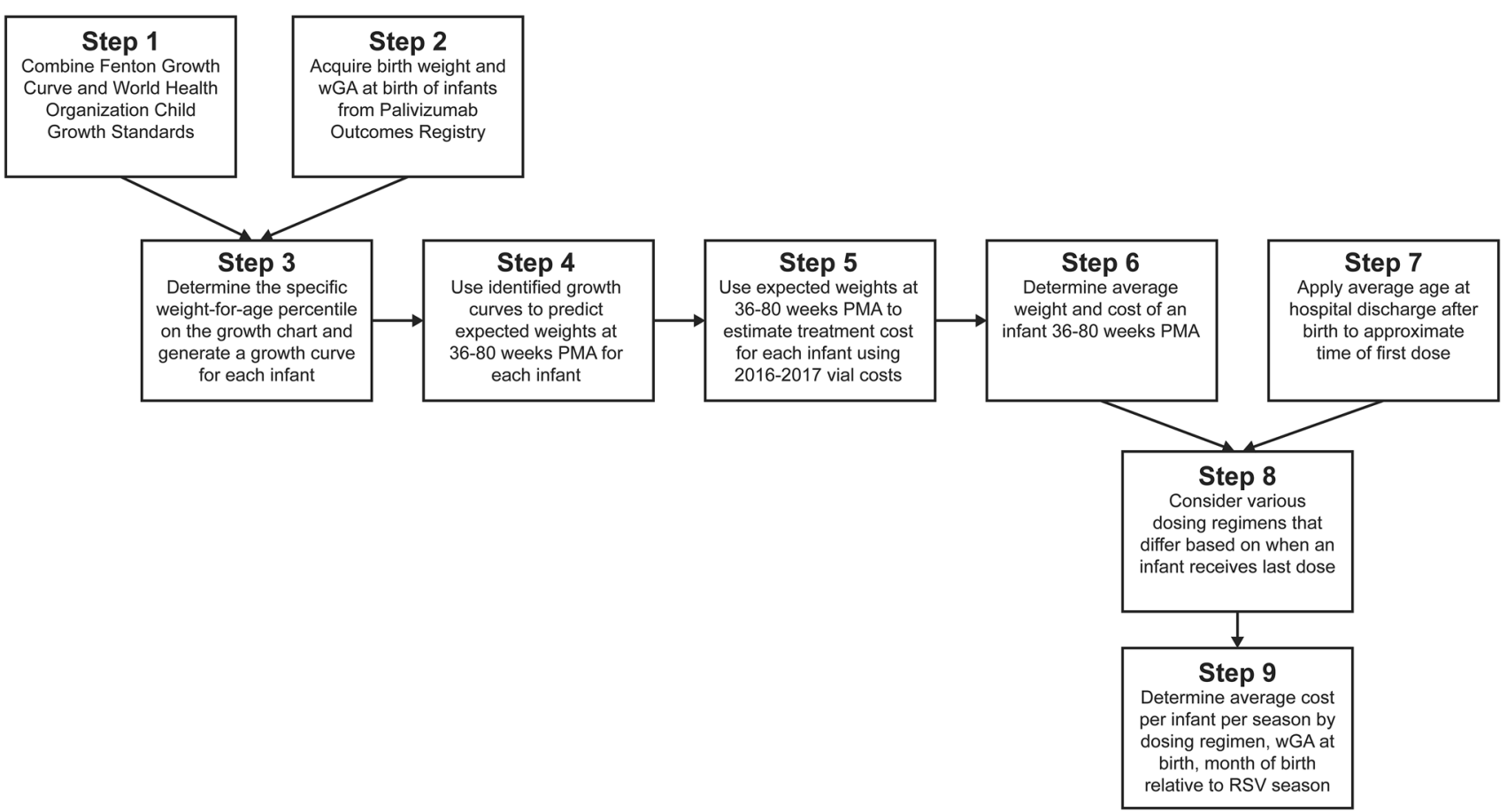

Fig. 1 Study approach in estimating mean costs of palivizumab per infant in the RSV season. $P M A$ postmenstrual age, $R S V$ respiratory syncytial virus, $w G A$ weeks gestational age

wGA with the WHO Child Growth Standards, which contain information on weight by week of chronological age. By linking the percentile curves from each growth chart, Fenton and Kim produced a growth chart beginning at 22 wGA and extending to 50 weeks postmenstrual age (PMA), defined as gestational age at birth plus chronological age.

In order to evaluate the growth of infants beyond 50 weeks PMA, we further extended the Fenton Growth Charts with the WHO Child Growth Standards for chronological age of 14 weeks (equivalent to 50 weeks PMA) and above. Because Fenton and Kim had used the WHO Child Growth Standards to produce the upper age range of their growth charts, we were able to seamlessly merge their growth curves with the WHO Child Growth Standards extending beyond 50 weeks PMA. Smoothing techniques were used to combine the curves while maintaining data integrity. Gender-specific curves were first created and then averaged in order to evaluate the growth of an average preterm infant.

Fenton Growth Charts measure age in weeks using the midpoint of a week, whereas the WHO Child Growth Standards measure age in complete weeks. Because the use of the midpoint of a week is preferable, the Fenton and WHO growth charts were combined in order to make daily, rather than weekly, predictions. Specifically, the LambdaMu-Sigma (LMS) parameters were smoothed from the Fenton and WHO growth charts over age using locally weighted scatter plot smoothing (LOESS) [15, 17]. Similarly, a span parameter was chosen that allowed original curve shapes to be maintained with a minimal degree of smoothing [15]. These daily LMS parameters were then converted into weekly parameters using the predicted estimates at day 3.5 during each week. Lastly, with LMS parameters in hand, we generated smoothed growth curves at any exact percentile.

\subsection{Using the Growth Chart to Determine Expected Weights of Infants from the Palivizumab Outcomes Registry}

The growth chart was used to determine the weight-for-age percentile at birth for any preterm infant and subsequently identify a growth curve specific to that infant. By following the growth curve, an infant's body weight can be estimated at any time, assuming the infant maintains the same growth pattern over time.

We applied this method to a sample of infants from the 2001-2002 RSV season of the Palivizumab Outcomes Registry-a US prospective, observational registry of children who received one dose or more of palivizumab for RSV prophylaxis from 2000 to 2004. The registry, extensively described in prior publications [18-20], collects data on demographics, clinical characteristics, and outcomes from a large sample of infants who received palivizumab, as well as more granular data such as exact birth weight for 
each infant, wGA at birth, and date of hospital discharge. Registry data were available for 5083 infants from the 2001-2002 RSV season.

The distribution of weight-for-age percentiles at birth for all infants in the 2001-2002 RSV season of the registry was very similar to the distribution of the subgroups of preterm infants born 29-32 and 33-35 wGA. Although preterm infants were born at an earlier gestational age and smaller weight, their percentiles of growth were distributed similarly to full-term infants. These similar distributions allowed all infants to be used in the next step [see the electronic supplementary material (ESM), online resource 1, Appendix Fig. A1].

The weight-for-age percentile for an infant was then identified on the basis of actual birth weight and wGA. After determining the birth weight-for-age percentile for each infant in the 2001-2002 RSV season, a growth curve was produced for each infant. The curve was used to determine infant weight at subsequent weekly time intervals by PMA.

\subsection{Estimating Cost of Treatment for an Infant}

Costs were estimated at each weekly PMA for all infants in the 2001-2002 RSV season and averaged for each age to derive expected mean costs of an infant by PMA. This approach allowed costs to be estimated at the individual level and averaged over the entire distribution of infants, instead of estimating costs for a typical (e.g., median) infant, in order to properly account for heterogeneity in costs across the population.

The calculated costs were based on the number of 50and 100-mg vials each infant required, based on the FDAapproved dose of $15 \mathrm{mg} / \mathrm{kg}$ of body weight, and the associated quarter 3 (Q3) 2016-Q2 2017 US wholesale acquisition costs of $\$ 1468.91$ for a 50-mg vial and \$2773.74 for a 100-mg vial (pricing data obtained from manufacturer).

\subsection{Estimating Mean Costs Under Various Dosing Regimens}

Expected costs by PMA were used to estimate average costs during the RSV season (November through March) under different real-world dosing regimen scenarios. The dosing regimens varied by the number of months after birth an infant could receive their last dose $(2,3,4$, or 5$)$, given that all infants start receiving treatment in the RSV season.

Two additional factors were considered before applying different dosing regimens: (1) uniform distribution of births throughout the year to ensure appropriate weighting is given to infants born in any given month; and (2) the estimated maximum PMA of first dose for an infant used to identify the start of treatment after birth.
To test the assumption that births are uniformly distributed throughout a given year, we examined the distribution of births from two different years, 2002 and 2014, using natality data from the National Center for Health Statistics [21]. Data separated by more than 10 years were chosen to test whether the distribution of births was maintained over time. The fraction of births within each month for the given year ranged from 0.08 to 0.09 for both years. Because the distribution of births was approximately uniform in both years, it is reasonable to assume uniform yearly birth distribution.

Using Palivizumab Outcomes Registry data from 2194 otherwise healthy preterm infants (29-35 wGA) admitted at birth to a neonatal intensive care unit, we derived an average age at discharge of 36 weeks PMA for preterm infants, which is an approximation of the maximum age of preterm infants for the first dose. Considering this age at discharge, hospital discharge occurred in the month after birth for preterm infants born 29-32 wGA and during the month of birth for preterm infants born 33-35 wGA.

Next, the cost of palivizumab was estimated for a preterm infant for each month of the 2016-2017 RSV season using the PMA at first dose, a given dosing regimen, and an RSV season spanning from November through March. Costs were calculated for all doses (dose at birth hospital discharge plus outpatient doses) and for outpatient doses only. To apply dosing regimens, we calculated how many months into the RSV season an infant born in a certain month was to be treated based on the dosing regimen. For example, in a dosing regimen of final dose given 2 months after birth, an infant born in September would receive her first and last injection in November. In the case of a fullseason scenario, infants born at any time of the year would receive five doses, starting in November. In each scenario, costs were a function of birth month and estimated age at birth hospital discharge. To calculate costs for a population given the earliest birth month included, the average cost at each PMA of expected dose for infants born in the earliest birth month and all costs in the following months were added and averaged by the number of months from the starting birth month to end of season (i.e., March) (Table 1). Subsequent doses after discharge injection occurred on a monthly basis according to FDA-approved dosing.

\subsection{Analysis of Variance}

After deriving average costs per infant, analysis of variance (ANOVA) was used to examine the key drivers of costs by determining the variables that best explain the variation in costs. ANOVA was performed using average cost as the outcome variable and birth month, dosing regimen, wGA at birth, interaction between dosing regimen and birth month, 
Table 1 Postmenstrual age at each expected dose of palivizumab for infants 29-32 and 33-35 wGA, based on birth month relative to the RSV season

\begin{tabular}{|c|c|c|c|c|c|c|c|c|c|c|}
\hline \multirow[t]{3}{*}{ Birth month } & \multicolumn{5}{|c|}{ PMA at dose, infants $29-32$ wGA } & \multicolumn{5}{|c|}{ PMA at dose, infants $33-35$ wGA } \\
\hline & \multicolumn{5}{|c|}{ RSV season } & \multicolumn{5}{|c|}{ RSV season } \\
\hline & Nov & Dec & Jan & Feb & Mar & Nov & Dec & Jan & Feb & Mar \\
\hline May & $56^{*}$ & 60 & 64 & 68 & 72 & $60^{*}$ & 64 & 68 & 72 & 76 \\
\hline Jun & $52^{*}$ & 56 & 60 & 64 & 64 & $56^{*}$ & 60 & 64 & 68 & 72 \\
\hline Jul & $48^{*}$ & 52 & 56 & 60 & 64 & $52^{*}$ & 56 & 60 & 64 & 68 \\
\hline Aug & $44^{*}$ & 48 & 52 & 56 & 60 & $48^{*}$ & 52 & 56 & 60 & 64 \\
\hline Sep & $40^{*}$ & 44 & 48 & 52 & 56 & $44^{*}$ & 48 & 52 & 56 & 60 \\
\hline Oct & $36^{*}$ & 40 & 44 & 48 & 52 & $40^{*}$ & 44 & 48 & 52 & 56 \\
\hline Nov & $x$ & $36^{*}$ & 40 & 44 & 48 & $36^{*}$ & 40 & 44 & 48 & 52 \\
\hline Dec & - & $x$ & $36^{*}$ & 40 & 44 & - & $36^{*}$ & 40 & 44 & 48 \\
\hline Jan & - & - & $x$ & $36^{*}$ & 40 & - & - & $36^{*}$ & 40 & 44 \\
\hline Feb & - & - & - & $\times$ & $36^{*}$ & - & - & - & $36^{*}$ & 40 \\
\hline Mar & - & - & - & - & $\times$ & - & - & - & - & $36^{*}$ \\
\hline
\end{tabular}

Months in bold indicate RSV season. Numbers with asterisks represent the PMA of the first dose for each given birth month. Crosses indicate that no dose would be provided in the month of birth because an infant was not of age to receive the first dose (PMA of 36 weeks or approximate age of hospital discharge after birth)

$P M A$ postmenstrual age, $R S V$ respiratory syncytial virus, $w G A$ weeks gestational age and interaction between dosing regimen and wGA at birth as independent variables. We then estimated how much each variable explained the variance (variation from the mean), in average cost per infant, by estimating their fraction of total variance (ratio of partial to total sums of squares for each variable). Additionally, to assess the quantitative relationship between the variables and the outcome, we used a linear regression model with average cost as the outcome and the same variables included in the ANOVA as the independent variables.

\subsection{Sensitivity Analyses}

Two real-world scenarios that could further impact the variability of treatment costs were considered in sensitivity analyses. The estimations for each of the sensitivity analyses were based on the same variables included in the main analysis. The first scenario allowed infants to receive a lower dose of palivizumab if their body weight was $\leq 10 \%$ higher than the weight required for the recommended dose. Palivizumab is supplied in 50- and 100-mg vials, and standard dosing assumes wastage; for example, an infant weighing $\leq 3.3 \mathrm{~kg}$ would receive a $50-\mathrm{mg}$ vial, but an infant weighing $3.4 \mathrm{~kg}$ would receive a $100-\mathrm{mg}$ vial. Dosing within $10 \%$ of body weight allows an infant at $3.4 \mathrm{~kg}$ to receive the $50-\mathrm{mg}$ vial instead of the $100-\mathrm{mg}$ vial, thereby impacting the dose cost for that infant. The second scenario considered post-acquisition manufacturer rebates, such as those required by federal law for Medicaid recipients [22, 23]. The average cost of palivizumab for Medicaid-insured preterm infants was estimated using a manufacturer rebate of $60 \%$ for Q3 2016 (rebate data obtained from manufacturer).

\section{Results}

Table 1 shows the PMAs at potential prophylactic administration times used for calculating costs for infants born 29-32 and 33-35 wGA. For each birth month, the PMA at expected dose for an infant is shown by month in the RSV season. The first dose of palivizumab was based on an average age of discharge of 36 weeks PMA. Infants 29-32 wGA born in the RSV season do not reach their maximum age for first dose in the same month they are born; therefore no dose would be provided in the birth month (represented by crosses in Table 1). Infants 33-35 wGA born in the $\mathrm{RSV}$ season reach their maximum age for first dose in the birth month. The included PMAs were customized for each dosing regimen.

The average treatment cost of palivizumab per infant per season based on various dosing regimens is presented in Table 2 for infants 29-32 and 33-35 wGA. Using the expected weight and cost at each PMA of palivizumab administration (Table 1), the average costs per infant were estimated given the dosing regimen, birth month relative to RSV season, and outpatient doses after first dose at birth discharge. The average costs of birth hospital discharge dose combined with outpatient doses were evaluated versus costs of outpatient doses only. Depending on the choice of dosing regimen, the estimated mean 2016-2017 total seasonal treatment cost of palivizumab for a preterm infant 
Table 2 Average seasonal cost of palivizumab (2016-2017 RSV season) for a preterm infant who receives treatment November-March, based on gestational age, birth month, and different dosing regimens

\begin{tabular}{|c|c|c|c|c|c|c|c|c|}
\hline & \multicolumn{2}{|c|}{$\begin{array}{l}\text { Last dose received } \\
2 \text { months after birth, } \\
\text { treatment starting } \\
\text { within RSV season }\end{array}$} & \multicolumn{2}{|c|}{$\begin{array}{l}\text { Last dose received } \\
3 \text { months after birth, } \\
\text { treatment starting } \\
\text { within RSV season }\end{array}$} & \multicolumn{2}{|c|}{$\begin{array}{l}\text { Last dose received } \\
4 \text { months after birth, } \\
\text { treatment starting } \\
\text { within RSV season }\end{array}$} & \multicolumn{2}{|c|}{$\begin{array}{l}\text { Treated monthly for } \\
\text { the full season (Nov- } \\
\text { Mar) }\end{array}$} \\
\hline & $\mathrm{BDD}+\mathrm{OD}$ & $\begin{array}{l}\text { OD } \\
\text { only }\end{array}$ & $\mathrm{BDD}+\mathrm{OD}$ & $\begin{array}{l}\text { OD } \\
\text { only }\end{array}$ & $\mathrm{BDD}+\mathrm{OD}$ & $\begin{array}{l}\text { OD } \\
\text { only }\end{array}$ & $\mathrm{BDD}+\mathrm{OD}$ & $\begin{array}{l}\text { OD } \\
\text { only }\end{array}$ \\
\hline \multicolumn{9}{|l|}{ Infants 29-32 wGA } \\
\hline Born in season (Nov-Mar) & $\$ 3287$ & $\$ 1733$ & $\$ 4664$ & $\$ 3110$ & $\$ 5370$ & $\$ 3816$ & $\$ 5370$ & $\$ 3816$ \\
\hline $\begin{array}{l}\text { Born in season }+<1 \text { month at season start } \\
\text { (Oct-Mar) }\end{array}$ & $\$ 3403$ & $\$ 1849$ & $\$ 5055$ & $\$ 3501$ & $\$ 6185$ & $\$ 4631$ & $\$ 6782$ & $\$ 5229$ \\
\hline $\begin{array}{l}\text { Born in season }+<2 \text { months at season start } \\
\text { (Sep-Mar) }\end{array}$ & $\$ 3221$ & $\$ 1926$ & $\$ 5056$ & $\$ 3762$ & $\$ 6469$ & $\$ 5174$ & $\$ 8021$ & $\$ 6726$ \\
\hline $\begin{array}{l}\text { Born in season }+<3 \text { months at season start } \\
\text { (Aug-Mar) }\end{array}$ & $\$ 3221^{\mathrm{a}}$ & $\$ 1926^{\mathrm{a}}$ & $\$ 4727$ & $\$ 3618$ & $\$ 6341$ & $\$ 5231$ & $\$ 9105$ & $\$ 7995$ \\
\hline $\begin{array}{l}\text { Born in season }+<4 \text { months at season start } \\
\text { (Jul-Mar) }\end{array}$ & $\$ 3221^{\mathrm{a}}$ & $\$ 1926^{\mathrm{a}}$ & $\$ 4727^{\mathrm{a}}$ & $\$ 3618^{\mathrm{a}}$ & $\$ 5902$ & $\$ 4931$ & $\$ 10,069$ & $\$ 9098$ \\
\hline $\begin{array}{l}\text { Born in season }+<5 \text { months at season start } \\
\text { (Jun-Mar) }\end{array}$ & $\$ 3221^{\mathrm{a}}$ & $\$ 1926^{\mathrm{a}}$ & $\$ 4727^{\mathrm{a}}$ & $\$ 3618^{\mathrm{a}}$ & $\$ 5902^{\mathrm{a}}$ & $\$ 4931^{\mathrm{a}}$ & $\$ 10,963$ & $\$ 10,100$ \\
\hline $\begin{array}{l}\text { Born in season }+<6 \text { months at season start } \\
\text { (May-Mar) }\end{array}$ & $\$ 3221^{\mathrm{a}}$ & $\$ 1926^{\mathrm{a}}$ & $\$ 4727^{\mathrm{a}}$ & $\$ 3618^{\mathrm{a}}$ & $\$ 5902^{\mathrm{a}}$ & $\$ 4931^{\mathrm{a}}$ & $\$ 11,799$ & $\$ 11,023$ \\
\hline \multicolumn{9}{|l|}{ Infants 33-35 wGA } \\
\hline Born in season (Nov-Mar) & $\$ 5055$ & $\$ 3501$ & $\$ 6185$ & $\$ 4631$ & $\$ 6782$ & $\$ 5229$ & $\$ 6782$ & $\$ 5229$ \\
\hline $\begin{array}{l}\text { Born in season }+<1 \text { month at season start } \\
\text { (Oct-Mar) }\end{array}$ & $\$ 5056$ & $\$ 3762$ & $\$ 6469$ & $\$ 5174$ & $\$ 7465$ & $\$ 6170$ & $\$ 8021$ & $\$ 6726$ \\
\hline $\begin{array}{l}\text { Born in season }+<2 \text { months at season start } \\
\text { (Sep-Mar) }\end{array}$ & $\$ 4727$ & $\$ 3618$ & $\$ 6341$ & $\$ 5231$ & $\$ 7622$ & $\$ 6512$ & $\$ 9105$ & $\$ 7995$ \\
\hline $\begin{array}{l}\text { Born in season }+<3 \text { months at season start } \\
\text { (Aug-Mar) }\end{array}$ & $\$ 4727^{\mathrm{a}}$ & $\$ 3618^{\mathrm{a}}$ & $\$ 5092$ & $\$ 4931$ & $\$ 7396$ & $\$ 6425$ & $\$ 10,069$ & $\$ 9098$ \\
\hline $\begin{array}{l}\text { Born in season }+<4 \text { months at season start } \\
\text { (Jul-Mar) }\end{array}$ & $\$ 4727^{\mathrm{a}}$ & $\$ 3618^{a}$ & $\$ 5902^{\mathrm{a}}$ & $\$ 4931^{\mathrm{a}}$ & $\$ 6907$ & $\$ 6043$ & $\$ 10,963$ & $\$ 10,100$ \\
\hline $\begin{array}{l}\text { Born in season }+<5 \text { months at season start } \\
\text { (Jun-Mar) }\end{array}$ & $\$ 4727^{\mathrm{a}}$ & $\$ 3618^{a}$ & $\$ 5902^{\mathrm{a}}$ & $\$ 4931^{\mathrm{a}}$ & $\$ 6907^{a}$ & $\$ 6043^{a}$ & $\$ 11,799$ & $\$ 11,023$ \\
\hline $\begin{array}{l}\text { Born in season }+<6 \text { months at season start } \\
\text { (May-Mar) }\end{array}$ & $\$ 4727^{\mathrm{a}}$ & $\$ 3618^{\mathrm{a}}$ & $\$ 5902^{\mathrm{a}}$ & $\$ 4931^{\mathrm{a}}$ & $\$ 6907^{\mathrm{a}}$ & $\$ 6043^{a}$ & $\$ 12,568$ & $\$ 11,862$ \\
\hline
\end{tabular}

$B D D$ birth discharge dose, $O D$ outpatient doses, $R S V$ respiratory syncytial virus, $w G A$ weeks gestational age

${ }^{a}$ Infants born in this earliest month were not eligible for dosing based on the specific policy. Therefore, the cost estimate reflected is equal to the earliest birth month eligible for the specific dosing policy

$<6$ months of age at the start of the RSV season or born during the season (May-March) ranged from \$3221 to $\$ 12,568$, with outpatient-only treatment costs between $\$ 1849$ and $\$ 11,862$. Costs for an infant born during the RSV season ranged from $\$ 3287$ to $\$ 6782$, with outpatientonly doses between $\$ 1733$ and $\$ 5229$.

Based on the sensitivity analyses, when considering $10 \%$ variation in body weight for dosing, average costs of treatment for a preterm infant $<6$ months of age at the start of the RSV season or born in the season ranged from $\$ 2847$ to $\$ 11,504$, with outpatient-only doses between $\$ 1530$ and $\$ 10,821$. For a preterm infant born only during the RSV season, the average costs ranged from $\$ 2938$ to $\$ 6313$, with outpatient-only doses between $\$ 1435$ and $\$ 4809$ (see the ESM, Appendix Table A1). Average costs of treatment for Medicaid-insured preterm infants, estimated using a manufacturer rebate of $60 \%$, are shown in Appendix Table A2 (see the ESM). Costs ranged from $\$ 1288$ to $\$ 5027$ for a preterm infant $<6$ months of age at the start of the RSV season or born during the season; outpatient-only costs ranged from $\$ 740$ to $\$ 4745$. For Medicaid-insured preterm infants born during the RSV season, the cost ranged from $\$ 1315$ to $\$ 2713$ (\$693 to \$2091 for outpatientonly doses).

Figure 2 shows the trend in expected weight by PMA from the derived growth chart used in the cost estimations. Appendix Fig. A2 (see the ESM) shows the average costs at each PMA for standard dosing, as well as the average 
Fig. 2 Average expected weight (kilograms) of an infant by postmenstrual age (PMA)

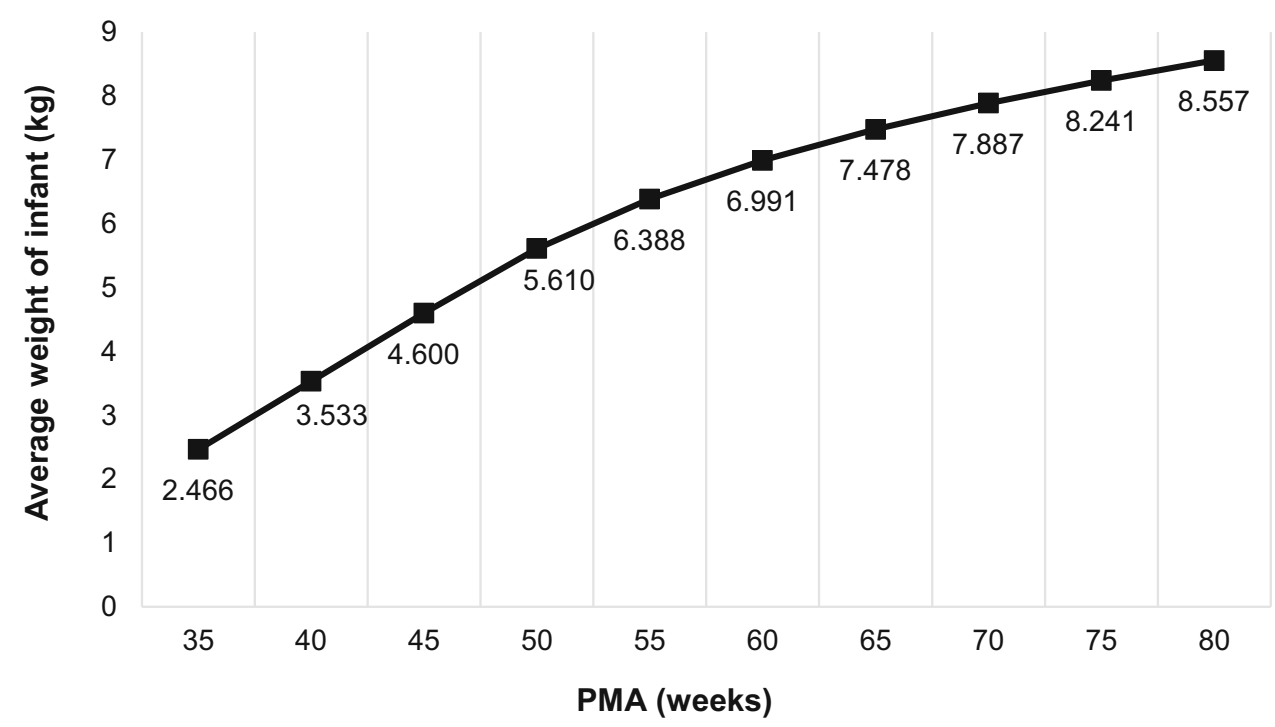

costs for infants who received a lower dose if their body weight was $\leq 10 \%$ higher than the weight required to receive the lower recommended dose. The difference in average per-infant cost between the two dosing approaches ranged from $\$ 18$ at 35 weeks PMA to $\$ 498$ at 61 weeks PMA. Appendix Fig. A2 (see the ESM) also shows the average costs at each PMA for Medicaid-insured infants, estimated using a $60 \%$ manufacturer rebate applied to standard dosing costs.

Although the expected average infant weight increased steadily with age (Fig. 2), average costs increased in a less consistent manner (see the ESM, Appendix Fig. A2). For standard dosing, the steepest incremental increase in cost between ages (i.e., differences in cost from one age to another) occurred at 37-41 weeks PMA, ranging from $\$ 130$ to $\$ 247$. For $35-36$ weeks PMA and $42-80$ weeks PMA, incremental cost for a 1-week difference in age varied from $\$ 11$ to $\$ 103$. Dosing within $10 \%$ variation of weight resulted in lower cost differences between ages. Under this scenario, the steepest incremental increase in cost between ages was seen among infants 38-43 weeks PMA.
Based on ANOVA, the key drivers of costs included dosing regimen (contributing to $74 \%$ of the variance in average cost for an infant, $p<0.0001)$, the interaction between dosing regimen and birth month (17\% of variance, $p<0.0001)$, wGA $(6 \%, p<0.0001)$, and birth month (3\% of variance, $p<0.0001$ ) (Table 3). Results from the linear regression model show that there is a positive effect on cost with the increase in number of months after birth an infant receives their last dose, a negative effect as birth month approaches the start of the RSV season (November), and a positive effect among infants 33-35 wGA at birth compared with those 29-32 wGA at birth [adjusted $R^{2}=0.9908$; standard error of regression (root-meansquare error) $=225.03$; see the ESM, Appendix Table A3].

\section{Discussion}

This study estimated the treatment costs of palivizumab for otherwise healthy preterm infants 29-35 wGA under several real-world scenarios. The analyses considered a range of potential dosing regimens in addition to estimating

Table 3 Key drivers of average cost for the RSV season: results from analysis of variance (ANOVA)

\begin{tabular}{lrrrr}
\hline Potential drivers of cost & $d f$ & $F$ statistic & $\begin{array}{c}P \text { value } \\
\text { Attributable fraction of variance of } \\
\text { average cost per infant }\end{array}$ \\
\hline Dosing regimen & 3 & 1464.41 & $<0.0001$ & 0.736 \\
Gestational age at birth (29-32 or 33-35 weeks) & 1 & 355.36 & $<0.0001$ & 0.060 \\
Birth month & 6 & 33.89 & $<0.0001$ & 0.034 \\
Interaction of dosing regimen and birth month & 18 & 55.47 & $<0.0001$ & 0.167 \\
Interaction of dosing regimen and gestational age at birth & 3 & 6.92 & $<0.005$
\end{tabular}

$R S V$ respiratory syncytial virus

${ }^{\text {a }}$ Calculated as ratio of partial sum of squares to the total sum of squares of the model 
infant growth over time, using a combination of the Fenton Growth Charts and WHO Child Growth Standards to more accurately calculate the cost at each prophylactic administration for an infant. The expected standard dosing costs of treating preterm infants 29-35 wGA at birth and $<6$ months of age at the start of the 2016-2017 RSV season or born in the season (May-March) ranged from $\$ 3221$ to $\$ 12,568$, with an outpatient treatment cost between $\$ 1733$ and $\$ 11,862$. When considering potential real-world scenarios, such as dose adjustments based on slight variation in body weight and manufacturer rebates for Medicaid-insured infants, further variation was observed, as standard dosing costs ranged between $\$ 1288$ and $\$ 11,504$ and outpatient-only dosing costs ranged from $\$ 740$ to $\$ 10,821$ for an infant $<6$ months of age at the start of the season or born in the season.

The results suggest considerable variability in the cost of treatment with palivizumab, mainly driven by choice of dosing regimen, which dictates the number of doses an infant can receive, in addition to infant's birth month, wGA, and weight-for-age, which drive the cost at each dose. The choice of dosing policy contributed to most of the variability in the average cost per infant per season, followed by the dosing policy's interacted with birth month, and wGA. This variability points to the potential of policies for RSV immunoprophylaxis to reduce the burden of disease by targeting utilization in the population with the greatest risk of severe RSV and lowest palivizumab cost, i.e., preterm infants during the first 6 months of life [24-29].

Previous cost-effectiveness studies made several assumptions of palivizumab cost and did not account for key cost drivers-particularly the different policy scenarios that contribute greatly to cost heterogeneity [8-11]. In general, studies assumed standard dosing (maximum number of doses, vial wastage), and uniform implementation of dosing guidelines, with the majority using a single estimate of infant weight across ages and doses. Therefore, it is likely that previous studies did not accurately estimate variation in costs across the population.

This analysis used real-world data from several data sources relevant to otherwise healthy preterm infants born 29-35 wGA to examine the effect of dosing policy variations on the cost of palivizumab in preterm infants. Further, it contributes to the existing literature by accounting for infant growth using weight-for-age when calculating cost, and provides a foundation for future research. From a policy perspective, this study highlights the importance of applying context when estimating real-world cost-effectiveness of technologies. Given the variation in costs and the role of key cost drivers, it is essential to avoid oversimplification of costing models that risk distorting realworld results.
It should be noted that this study did not factor in the real-world practice of vial sharing (splitting the contents of a single-use vial to provide the drug to more than one eligible infant), or price decreases due to potential future patent expiry, so these estimates will be at the high end of the real-world costs per infant. The results of this study are applicable to the otherwise healthy preterm population of infants born 29-35 wGA, and may not be generalizable to other high-risk infant groups. Further, this study assumed average maximum age for first dose for all preterm infants to be time of birth hospital discharge, as initial dosing is recommended to occur at this time according to AAP guidelines.

\section{Conclusion}

From a public health standpoint, the healthcare burden of severe RSV in preterm infants is reduced with immunoprophylaxis $[30,31]$. Because acquisition costs of palivizumab in cost-effectiveness studies are tools used to develop policy guidelines, it is crucial to evaluate costs based on different real-world scenarios that are key drivers of cost in order to provide a more accurate picture of the economic and health benefits. This study found considerable variability in the average cost of palivizumab treatment for otherwise healthy preterm infants born 29-35 wGA, driven by choice of dosing regimen, wGA, and birth month. It also highlights the importance of considering patient heterogeneity and context in understanding the often considerable variance that exists for inputs in palivizumab cost-effectiveness models. Making policy decisions based on cost-effectiveness estimates for an "average" patient is dangerous when the goal is health gain optimization for a population or a particular set of patients in need. Therefore, future studies should consider both weight-for-age at dosing and dosing policies in order to provide more accurate assessments of cost-effectiveness.

Acknowledgements Manuscript preparation support was provided by The Lockwood Group, which was in accordance with Good Publication Practice (GPP3) and funded by AstraZeneca.

Author contributions Study concept and design were contributed to by AS, DI, DP, KM, and WS. AS and DI collected and analyzed the data. Data interpretation was performed by AS, DI, DP, KM, and WS. The manuscript was written and revised by AS, DI, DP, KM, and WS.

\section{Compliance with Ethical Standards}

Conflict of interest AS, DI, DP, and WS are employees of Precision Health Economics, which received funding from AstraZeneca to conduct this work. KM is an employee of AstraZeneca. The funding agreement ensured the authors' independence in designing the study, interpreting the data, and writing the manuscript. 
Funding This study was supported by AstraZeneca.

Data availability statement The Fenton Growth Charts that support the findings of this study are available from Dr. Tanis Fenton at the University of Calgary; however, restrictions apply to the availability of these data, which were used under license for the current study, and are therefore not publicly available. The Palivizumab Outcomes Registry data analyzed during the current study are not publicly available. The WHO Child Growth Standards (http://www.who.int/ childgrowth/standards/en/) and the National Center for Health Statistics natality birth data (http://www.nber.org/data/vital-statisticsnatality-data.html) are publicly available data and are available from the corresponding author upon reasonable request.

Open Access This article is distributed under the terms of the Creative Commons Attribution-NonCommercial 4.0 International License (http://creativecommons.org/licenses/by-nc/4.0/), which permits any noncommercial use, distribution, and reproduction in any medium, provided you give appropriate credit to the original author(s) and the source, provide a link to the Creative Commons license, and indicate if changes were made.

\section{References}

1. Nair H, et al. Global burden of acute lower respiratory infections due to respiratory syncytial virus in young children: a systematic review and meta-analysis. Lancet. 2010;375(9725):1545-55.

2. Resch B, Paes B. Are late preterm infants as susceptible to RSV infection as full term infants? Early Human Dev. 2011;87:S47-9.

3. Gijtenbeek RG, et al. RSV infection among children born moderately preterm in a community-based cohort. Eur $\mathrm{J}$ Pediatr. 2015;174(4):435-42.

4. Lakdawalla D, et al. Quality-adjusted cost of care: a meaningful way to measure growth in innovation cost versus the value of health gains. Health Aff (Millwood). 2015;34(4):555-61.

5. Brady MT, et al. Updated guidance for palivizumab prophylaxis among infants and young children at increased risk of hospitalization for respiratory syncytial virus infection. Pediatrics. 2014;134(2):e620-38.

6. Bocchini JA, et al. Policy statement-modified recommendations for use of palivizumab for prevention of respiratory syncytial virus infections. Pediatrics. 2009;124(6):1694-701.

7. Gutfraind A, Galvani AP, Meyers LA. Efficacy and optimization of palivizumab injection regimens against respiratory syncytial virus infection. JAMA pediatr. 2015;169(4):341-8.

8. Yount LE, Mahle WT. Economic analysis of palivizumab in infants with congenital heart disease. Pediatrics. 2004;114(6):1606-11.

9. Wegner S, et al. Direct cost analyses of palivizumab treatment in a cohort of at-risk children: evidence from the North Carolina Medicaid Program. Pediatrics. 2004;114(6):1612-9.

10. Prescott WA Jr, et al. Cost effectiveness of respiratory syncytial virus prophylaxis. Pharmacoeconomics. 2010;28(4):279-93.

11. Weiner LB, et al. Cost-effectiveness analysis of palivizumab among pre-term infant populations covered by Medicaid in the United States. J Med Econ. 2012;15(5):997-1018.

12. Stevens W, Normand C. Optimisation versus certainty: understanding the issue of heterogeneity in economic evaluation. Soc Sci Med. 2004;58(2):315-20.

13. Espinoza MA, et al. The value of heterogeneity for cost-effectiveness subgroup analysis conceptual framework and application. Med Decis Making. 2014;34(8):951-64.
14. Basu A, et al. Heterogeneity in action: the role of passive personalization in comparative effectiveness research. Health Econ. 2014;23(3):359-73.

15. Fenton TR, Kim JH. A systematic review and meta-analysis to revise the Fenton growth chart for preterm infants. BMC Pediatr. 2013;13(1): 1

16. World Health Organization. The WHO Child Growth Standards. [cited 2016 August 30]. http://www.who.int/childgrowth/ standards/en/. Accessed 1 Mar 2016.

17. Flegal KM, Cole TJ. Construction of LMS parameters for the Centers for Disease Control and Prevention 2000 growth charts. Natl Health Stat Rep. 2013;63:1-4.

18. La Gamma EF, et al. Receipt of palivizumab before birth hospitalization discharge among preterm infants in the United States. Am J Perinatol. 2015;32(11):1017-23.

19. Frogel M, et al. Prevention of hospitalization due to respiratory syncytial virus: results from the Palivizumab Outcomes Registry. J Perinatol. 2008;28(7):511-7.

20. Parnes C, et al. Palivizumab prophylaxis of respiratory syncytial virus disease in 2000-2001: results from the Palivizumab Outcomes Registry. Pediatr Pulmonol. 2003;35(6):484-9.

21. National Center for Health Statistics (NCHS). NCHS' vital statistics natality birth data. 1968-2014. http://www.nber.org/ data/vital-statistics-natality-data.html. Accessed 23 Sep 2016.

22. Ambrose CS, McLaurin KK. The Medicaid cost of palivizumab. J Pediatr Infect Dis Soc. 2015;4(1):83-4.

23. Centers for Medicare and Medicaid Services. Unit rebate amount (URA) calculation for single source or innovator multiple source drugs. [cited 2016 November 21]. https://www.medicaid.gov/ medicaid-chip-program-information/by-topics/prescription-drugs/ downloads/ura-for-s-or-i.pdf. Accessed 11 Aug 2016.

24. Boyce TG, et al. Rates of hospitalization for respiratory syncytial virus infection among children in Medicaid. $\mathrm{J}$ Pediatr. 2000;137(6):865-70.

25. Anderson EJ, et al. SENTINEL1: an observational study of respiratory syncytial virus hospitalizations among US infants born at 29 to 35 weeks' gestational age not receiving immunoprophylaxis. Am J Perinatol. 2017;34(1):51-61.

26. Winterstein AG, et al. Appropriateness of age thresholds for respiratory syncytial virus immunoprophylaxis in moderate-preterm infants: a cohort study. JAMA Pediatr. 2013;167(12): $1118-24$.

27. Helfrich AM, et al. Healthy Late-preterm infants born $33-36+6$ weeks gestational age have higher risk for respiratory syncytial virus hospitalization. Early Human Dev. 2015;91(9): 541-6.

28. Ambrose CS, et al. Respiratory syncytial virus disease in preterm infants in the US born at 32-35 weeks gestation not receiving immunoprophylaxis. Pediatr Infect Dis J. 2014;33(6):576-82.

29. Simões EA, et al. Effects of chronologic age and young child exposure on respiratory syncytial virus disease among US preterm infants born at 32 to 35 weeks gestation. PLoS One. 2016;11(11):e0166226.

30. The IMpact-RSV Study Group. Palivizumab, a humanized respiratory syncytial virus monoclonal antibody, reduces hospitalization from respiratory syncytial virus infection in high-risk infants. Pediatrics. 1998;102(3):531-7.

31. Blanken MO, et al. Prospective validation of a prognostic model for respiratory syncytial virus bronchiolitis in late preterm infants: a multicenter birth cohort study. PLoS One. 2013;8(3): e59161. 